\title{
Quantum geometry of chiral bosons on a circle
}

\author{
J. R. Reyes Martinez and A. H. Zimerman \\ Instituto de Física Teórica, Universidade Estadual Paulista, Rua Pamplona 145, 01405 São Paulo, São Paulo, Brazil \\ P. Teotônio Sobrinho \\ Instituto de Física, Universidade de São Paulo, Caixa Postal 20516, 01498 São Paulo, São Paulo, Brazil
}

(Received 24 October 1988)

\begin{abstract}
We extend the geometric treatment done for the Majorana-Weyl fermions in two dimensions by Sanielevici and Semenoff to chiral bosons on a circle. For this case we obtain a generalized Floreanini-Jackiw Lagrangian density, and the corresponding gravitational (or Virasoro) anomalies are found as expected.
\end{abstract}

\section{INTRODUCTION}

Studying the Majorana-Weyl fermions in two dimensions Sanielevici and Semenoff, ${ }^{1}$ by an explicit construction, connected the curvature of a fiber bundle constructed on the space of reparametrizations $M=\operatorname{Diff} S^{1} / S^{1}$ with the gravitational anomaly of these fermions in a static background field. This construction was inspired by the work of Bowick and Rajeev ${ }^{2}$ who have complexified the space $M$ and introduced in this way a Kähler connection. They showed that the anomaly of the Virasoro algebra is just the corresponding curvature of a vector bundle over complex $M$.

In their work Sanielevici and Semenoff give an explicit construction of the fiber bundle made by the Hilbert space of the fermion states in a background metric field. The purpose of our paper is to extend their method to chiral bosons on a circle and at the same time to make an elementary discussion of the geometry of the problem.

In Sec. I we introduce chiral bosons on a circle, discuss the corresponding Dirac constraints, and obtain the generalization of the Floreanini-Jackiw ${ }^{3}$ Lagrangian density for periodic and antiperiodic boundary conditions.

In Sec. II we introduce in an elementary way the group of reparametrizations Diff $S^{1} / S^{1}$ and obtain the Hamiltonian for each point of this space. We find that the structure of the phase space (sympletic form) is invariant under reparametrizations. The system is quantized and the corresponding Fock spaces are introduced.

In Sec. III the quantum geometry of the chiral bosons in a static external background field is discussed and a connection with curvature is introduced as well as the corresponding adiabatic quantities.

In Sec. IV the Hamiltonian of the chiral bosons in a static external background is rewritten in terms of the bosonic field. We make a little remark regarding the work of Bellucci, Golterman, and Petcher, ${ }^{4}$ and extend to this case their treatment introduced by Sanielevici and Semenoff. ${ }^{3}$

In Sec. $\mathrm{V}$ we calculate the curvature for chiral bosons on the circle by extending to this case the methods of Ref. 3 and discuss its relation with the curvature intro- duced in Sec. III. Some short details of the relevant calculations are given in the appendixes.

\section{CHIRAL BOSONS ON A CIRCLE}

Let us consider chiral bosons on a circle of length $2 \pi$ described by the Lagrangian density

$$
\mathcal{L}=\frac{1}{2}\left(\partial_{\tau} \phi\right)^{2}-\frac{1}{2}\left(\partial_{\alpha} \phi\right)^{2}
$$

subject to the constraint

$$
\left(\partial_{\tau} \phi-\partial_{\alpha} \phi\right) \approx 0 \text {. }
$$

The Hamiltonian density corresponding to (1.1) is

$$
\mathscr{H}=\frac{1}{2} \Pi^{2}(\alpha)+\frac{1}{2} \phi^{\prime 2}(\alpha) .
$$

We shall first consider periodic boundary conditions

$$
\phi(\alpha)=\phi(\alpha+2 \pi) \text {. }
$$

by introducing the variables

$$
\begin{aligned}
& T^{R}(\alpha)=\frac{1}{\sqrt{2}}\left[\pi(\alpha)-\phi^{\prime}(\alpha)\right], \\
& T^{L}(\alpha)=\frac{1}{\sqrt{2}}\left[\pi(\alpha)+\phi^{\prime}(\alpha)\right],
\end{aligned}
$$

and the corresponding components

$$
T_{n}^{L, R}=\int \varphi_{n}^{*}(\alpha) T^{L, R}(\alpha) d \alpha,
$$

where

$$
\varphi_{n}(\alpha)=\frac{1}{\sqrt{2}} \exp (\text { in } \alpha)
$$

In the above relations the "time" $\tau$ is fixed, $\phi^{\prime}(\alpha)=d \phi(\alpha) / d \alpha$ and $n \in \mathbb{Z}$. We can show that $\Pi(\alpha)$, $\phi(\alpha)$, and the total Hamiltonian can be rewritten in terms of $T_{n}^{R}$ and $T_{n}^{L}$ as

$$
\Pi(\alpha)=\frac{1}{\sqrt{2}} \sum_{n}\left(T_{n}^{L}+T_{n}^{R}\right) \varphi_{n}(\alpha),
$$


$\phi(\alpha)=\frac{1}{\sqrt{2}}\left[\sum_{n \neq 0} \frac{-i}{n}\left(T_{n}^{L}-T_{n}^{R}\right) \varphi_{n}(\alpha)+\frac{\phi_{0}}{\sqrt{2 \pi}}\right]$

$H=\frac{1}{2} \sum_{n}\left(T_{n}^{L} T_{-n}^{L}+T_{n}^{R} T_{-n}^{R}\right)$,

where $\phi_{0}$ is a constant of integration in $\alpha$.

By imposing the Poisson brackets

$$
\begin{aligned}
& \left\{\Pi(\alpha), \phi\left(\alpha^{\prime}\right)\right\}=-\delta\left(\alpha-\alpha^{\prime}\right), \\
& \left\{\phi(\alpha), \phi\left(\alpha^{\prime}\right)\right\}=\left\{\Pi(\alpha), \Pi\left(\alpha^{\prime}\right)\right\}=0
\end{aligned}
$$

we arrive at the Poisson brackets

$$
\begin{aligned}
& \left\{T_{n}^{L}, T_{m}^{L}\right\}=i n \delta_{n+m, 0}, \\
& \left\{T_{n}^{R}, T_{m}^{R}\right\}=-i n \delta_{n+m, 0}, \\
& \left\{T_{n}^{L}, T_{m}^{R}\right\}=0, \\
& \left\{\phi_{0}, T_{n}^{L}\right\}=0, \\
& \left\{\phi_{0}, T_{n}^{R}\right\}=0, \quad n \neq 0, \\
& \left\{\phi_{0}, T_{0}\right\}=1,
\end{aligned}
$$

where $T_{0}=T_{0}^{R}=T_{0}^{L}$. The constraint (1.2), in terms of the components $T_{n}$, can be rewritten as

$$
T_{n}^{R} \approx 0
$$

for all integers $n$. The algebra of the constraints is described by the relations (1.14). Let us note that the constraint in time of (1.19) does not give any new secondary constraints. In particular (1.14) shows that $T_{0}$ is a firstclass constraint while $T_{n}^{R}$ are second-class constraints (for $n \neq 0$ ). Also (1.18) shows that the constraint $T_{0} \approx 0 \mathrm{im}-$ plies that our system does not depend on $\phi_{0}$, that is, $\phi(\alpha) \rightarrow \phi(\alpha)+$ const is a symmetry of our problem. Therefore, we can choose the "gauge" $\phi_{0} \approx 0$ and the constraint $T_{0} \approx 0$ becomes a second-class one. In this way we remain with only second-class constraints. By following the Dirac method we define new Poisson brackets and now the constraints become strong equations and they disappear from the formalism. With the definition of the Dirac brackets: ${ }^{5}$

$$
\begin{aligned}
\{A, B\}_{D}= & \{A, B\}-\sum_{n \neq 0}\left\{A, T_{-n}^{R}\right\} \frac{1}{i n}\left\{T_{n}^{R}, B\right\} \\
& -\left\{A, \phi_{0}\right\}\left\{T_{0}, B\right\}-\left\{A, T_{0}\right\}\left\{\phi_{0}, B\right\}
\end{aligned}
$$

it follows that

$$
\begin{aligned}
& \left\{\Pi(\alpha), \Pi\left(\alpha^{\prime}\right)\right\}=\frac{1}{2} \partial_{\alpha} \delta\left(\alpha-\alpha^{\prime}\right), \\
& \left\{\phi(\alpha), \Pi\left(\alpha^{\prime}\right)\right\}=\frac{1}{2} \delta\left(\alpha-\alpha^{\prime}\right), \\
& \left\{\phi(\alpha), \phi\left(\alpha^{\prime}\right)\right\}=-\frac{1}{4} \epsilon\left(\alpha-\alpha^{\prime}\right),
\end{aligned}
$$

where all the brackets are the Dirac ones. We have defined the functions $\delta\left(\alpha-\alpha^{\prime}\right)$ and $\epsilon\left(\alpha-\alpha^{\prime}\right)$ by means of

$$
\begin{aligned}
& \delta\left(\alpha-\alpha^{\prime}\right)=\sum_{n \neq 0} \varphi_{n}(\alpha) \varphi_{-n}(\alpha), \\
& \epsilon\left(\alpha-\alpha^{\prime}\right)=2 \sum_{n \neq 0} \frac{1}{i n} \varphi_{n}(\alpha) \varphi_{-n}(\alpha) .
\end{aligned}
$$

We can now define real canonical variables

$$
\begin{aligned}
& q_{n}=\frac{1}{\sqrt{2}}\left(T_{n}^{L}+T_{-n}^{L}\right), \\
& p_{n}=\frac{i}{\sqrt{2} n}\left(T_{n}^{L}-T_{-n}^{L}\right)
\end{aligned}
$$

for $n>0$ with

$$
\left\{q_{n}, p_{m}\right\}=\delta_{n, m} .
$$

So the total Lagrangian is given as usual by

$$
L=\sum_{n \neq 0} p_{n} \dot{q}_{n}-H\left(q_{n}, p_{n}\right),
$$

where $H\left(q_{n}, p_{n}\right)$ is obtained from (1.11), remembering that now we have $T_{n}^{R}=0$ strongly. We obtain from (1.9), (1.10), (1.26), and (1.27) the Lagrangian in terms of the original field (with $T_{n}^{R}=0$ ):

$$
L=\frac{1}{2} \int d \alpha d \alpha^{\prime} \Pi(\alpha) \epsilon\left(\alpha-\alpha^{\prime}\right) \dot{\Pi}\left(\alpha^{\prime}\right)-\int d \sigma \Pi^{2}(\alpha) .
$$

We can redefine $\phi \rightarrow(1 / \sqrt{2}) \phi$ and $\Pi \rightarrow(1 / \sqrt{2}) \Pi$ to get

$$
L=\frac{1}{4} \int d \alpha d \alpha^{\prime} \Pi(\alpha) \epsilon\left(\alpha-\alpha^{\prime}\right) \dot{\Pi}\left(\alpha^{\prime}\right)-\frac{1}{2} \int d \alpha \Pi^{2}(\alpha),
$$

which is a generalization for the circle of the form given by Floreanini and $\mathrm{Jackiw}^{3}$ for case of whole real axis.

In our case $\epsilon(x)$ has the graphical representation given in Fig. 1.

In terms of $\phi(\alpha)$ expression (1.30b), recalling that $\Pi(\alpha)=\phi^{\prime}(\alpha)$, one gets

$$
L=\int d \sigma \frac{1}{2}\left[\phi^{\prime}(\alpha) \dot{\phi}(\sigma)-\phi^{\prime 2}(\alpha)\right],
$$

which is again similar to the one given by Floreanini and Jackiw. $^{3}$

For antiperiodic boundary conditions

$$
\phi(\alpha)=-\phi(\alpha+2 \pi),
$$

the treatment is similar with exception that instead of $n \in \mathbb{Z}$ we have $n \in \mathbb{Z}+\frac{1}{2}$ in expression (1.8) and all that which follow. In this case we do not need to discuss zero modes since they do not appear.

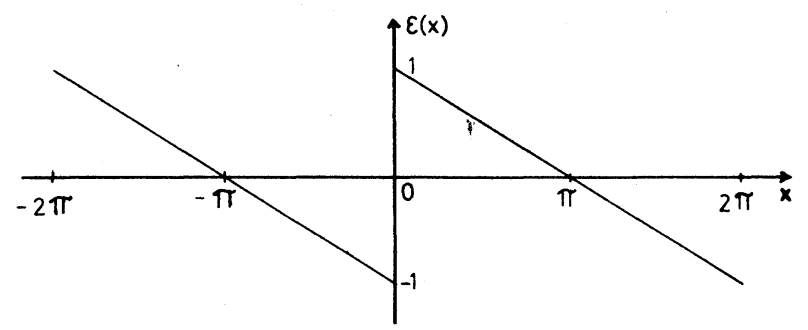

FIG. 1. Graphic representation of $\epsilon(x)$. 


\section{CHIRAL BOSONS IN A CURVED SPACE}

Let us now introduce a length parameter $L(L=2 \pi$ in the circular case) of periodicity:

$$
\phi(\alpha+L, \tau)=\phi(\alpha, \tau)(-1)^{S},
$$

where $S=0$ for periodic boundary conditions (PBC's), $S=1$ for antiperiodic boundary conditions (ABC's). Instead of (1.8) we have

$$
\begin{aligned}
& \varphi_{n}(\alpha)=\frac{1}{\sqrt{L}} \exp \left[i \frac{2 \pi}{L} n \alpha\right], \\
& n \in \mathbb{Z} \text { for } \mathrm{PBC}, \quad n \in \mathbb{Z}+\frac{1}{2} \text { for } \mathrm{ABC}
\end{aligned}
$$

and the corresponding constrained system described by (1.1) and (1.2) follows similarly with the exception that on the right-hand side for (1.13) and (1.14) we have an additional factor $2 \pi / L$.

It is expected that the classical theory should be invariant under a change of coordinates in the variable $\alpha$ (reparametrizations):

$$
\alpha \rightarrow \alpha^{\prime}=\sigma(\alpha) \text {. }
$$

The space of all reparametrizations forms a group called DiffS ${ }^{1}$. If we impose that the classical theory is invariant under this group, we see that the fields must transform in the following way:

$$
\Pi^{(2)}\left(\sigma_{2}(\alpha)\right)=\frac{d \sigma_{1}}{d \sigma_{2}} \Pi^{(1)}\left(\sigma_{1}(\alpha)\right)
$$

or

$$
\Pi^{(2)}\left(\sigma_{2}(\alpha)\right)=\eta_{1}(\alpha) \eta_{2}^{-1}(\alpha) \Pi^{(1)}\left(\sigma_{1}(\alpha)\right),
$$

where

$$
\eta_{1}(\alpha)=\frac{d \sigma_{1}(\alpha)}{d \alpha}, \quad \eta_{2}(\alpha)=\frac{d \sigma_{2}(\alpha)}{d \alpha} .
$$

Also

$$
\phi^{(1)}\left(\sigma_{1}(\alpha)\right)=\phi^{(2)}\left(\sigma_{2}(\alpha)\right) .
$$

If we consider the fields expressed in terms of $T_{n}\left(=T_{n}^{L}\right.$ since $T_{n}^{R}=0$ ), they can be considered as vectors of the form $\left(. ., T_{-2}, T_{-1}, T_{1}, T_{2}, \ldots\right)$, by introducing the metric

$$
\begin{aligned}
& \eta^{m n}=\delta^{m+n, 0}, \\
& \eta_{m n}=\delta_{m+n, 0} .
\end{aligned}
$$

The Hamiltonian (1.11) can be rewritten

$$
H=\frac{1}{2} \eta^{m n} T_{m} T_{n}
$$

An "observer" which would use $\sigma(\alpha)$ in order to parametrize the circle instead of $\alpha$ would use the following functions for the Fourier expansion:

$$
\varphi^{n[\sigma]}(\alpha)=\varphi^{n}(\sigma(\alpha))=\frac{1}{\sqrt{L}} \exp \left[i \frac{2 \pi}{L} n \sigma(\alpha)\right],
$$

which satisfy

$$
\int_{0}^{2 \pi} d \alpha \eta(\alpha) \eta_{m k} \varphi^{n[\sigma]}(\alpha) \varphi^{k[\sigma]}(\alpha)=\delta_{m}^{n} .
$$

Therefore, if $f(\alpha+L)=f(\alpha)(-1)^{s}$ then

$$
f(\alpha)=f_{[\sigma] n} \varphi^{n[\sigma]}(\alpha),
$$

where

$$
f_{[\sigma] m}=\int d \alpha \eta(\alpha) f(\alpha) \eta_{m n} \varphi^{n[\sigma]}(\alpha) .
$$

Let us consider two reparametrizations $\sigma_{1}(\alpha)$ and $\sigma_{2}(\alpha)$. Then

$$
\begin{aligned}
& \Pi^{(1)}\left(\sigma_{1}(\alpha)\right)=T_{\left[\sigma_{1}\right] n} \varphi^{n\left[\sigma_{1}\right]}(\alpha), \\
& \Pi^{(2)}\left(\sigma_{2}(\alpha)\right)=T_{\left[\sigma_{2}\right] n} \varphi^{n\left[\sigma_{2}\right]}(\alpha) .
\end{aligned}
$$

By the law of transformation (2.5) it follows

$$
T_{\left[\sigma_{2}\right] n}=e_{\left[\sigma_{2}\right] n}^{\left[\sigma_{1}\right] m} T_{\left[\sigma_{1}\right] m},
$$

where

$$
e_{\left[\sigma_{2}\right] n}^{\left[\sigma_{1}\right] 1}=\eta_{n k} \int d \alpha \eta_{1}(\alpha) \varphi^{k\left[\sigma_{2}\right]}(\alpha) \varphi^{1\left[\sigma_{1}\right]}(\alpha) .
$$

Putting $\sigma_{2}=I$ (identity) and $\sigma_{2}=\sigma$ in (2.5), we have

$$
T_{[\sigma] n}=e_{[\sigma] n}^{m} T_{m} .
$$

Analogously

$$
T_{n}=e^{[\sigma] m} T_{[\sigma] m} \text {. }
$$

The Hamiltonian (2.9) can be rewritten as

$$
H=\frac{1}{2} \eta^{m n} e^{[\sigma] a} e^{[\sigma] b} T_{[\sigma] a} T_{[\sigma] b}
$$

or

$$
H=\varrho^{[\sigma] a b} T_{[\sigma] a} T_{[\sigma] b} .
$$

With the definition

$$
\varrho^{[\sigma] a b}=\frac{1}{2} \eta^{m n} e_{m}^{[\sigma] a} e_{n}^{[\sigma] b}
$$

we can see that $g^{[\sigma] a b}$ is not affected by a rigid transformation $\sigma(\alpha)=\alpha+k$. Therefore, we consider only the quotient space DiffS ${ }^{1} / S^{1}$.

As we have

$$
\begin{gathered}
e^{[\sigma] a} e_{[\sigma] a}^{n}=\delta_{m}^{n}, \\
e^{[\sigma] a} e_{[\sigma] b}^{m}=\delta_{b}^{a}
\end{gathered}
$$

we can define an inverse

$$
\mathcal{S}_{[\sigma] a b}=\eta_{m n} e_{[\sigma] a}^{m} e_{[\sigma] b}^{n} .
$$

Let us now calculate the Poisson brackets

$$
\begin{aligned}
\left\{T_{[\sigma] n}, T_{[\sigma] m}\right\} & =-e_{[\sigma] n}^{l} e_{[\sigma] m}^{k}\left\{T_{l}, T_{k}\right\} \\
& =-i \sum\left(\frac{2 \pi}{L}\right) l e_{[\sigma] n}^{-l} e_{[\sigma] m}^{l}
\end{aligned}
$$

which by (2.17) gives

$$
\left\{T_{[\sigma] n}, T_{[\sigma] m}\right\}=\operatorname{in}\left[\frac{2 \pi}{L}\right] \delta_{n+m, 0} .
$$


We see, therefore, that the Poisson brackets (2.26) do not depend on the parameter $\sigma$ which is used. This is nothing more than the covariance of the Poisson brackets (1.21), (1.22), and (1.23) under the action of DiffS $S^{1} / S^{1}$ (reparametrizations). But the Hamiltonian (2.21) is not covariant (invariant in form) by a reparametrization. For each $\sigma \in \operatorname{Diff} S^{1} / S^{1}$ the form of $H$, that is, the metric, is different. The metric $\varrho^{[\sigma] a b}$ is not diagonal in general, but using (2.23) we can diagonalize it.

For every $\sigma \in \operatorname{Diff} S^{1} / S^{1}$ we can quantize our system canonically using the rule

$$
\{,\} \rightarrow \frac{1}{i}[,]
$$

and considering now $T_{[\sigma] n}$ as an operator. We have the commutation relations

$$
\left[T_{[\sigma] n}, T_{[\sigma] m}\right]=-n\left(\frac{2 \pi}{L}\right) \delta_{n+m, 0}
$$

and $T_{[\sigma] n}^{\dagger}=T_{[\sigma]-n}$.

Defining the operator

$$
a_{n}=e^{[\sigma] m} T_{[\sigma] m}\left(\frac{2 \pi}{L}\right)^{1 / 2}
$$

from relation (2.27) it follows that

$$
\left[a_{n}^{\dagger}, a_{m}\right]=\delta_{m+n, 0}, \quad n, m>0
$$

and the other being zero. The operators $a_{n}$ are creation operators while the $a_{n}^{\dagger}$ are destruction operators. The normal-ordered Hamiltonian is

$$
: H:=\sum_{\eta} \omega_{n} a_{n} a_{n}^{\dagger}
$$

with

$$
\omega_{n}=\frac{2 \pi n}{L} .
$$

The Fock space is constructed from the vacuum state (assuming not degenerate) defined by

$$
a_{n}^{\dagger}|0\rangle=0 \text {. }
$$

By successive application of the operator $a_{n}$ on the vacuum we obtain the states for $1,2,3, \ldots$ particles generating the Fock space. For each $\sigma \in \operatorname{DiffS}^{1} / S^{1}$ we construct a Fock space and all these are isomorphic between them since the commutation relations do not depend on $\sigma$.

\section{QUANTUM GEOMETRY OF THE CHIRAL BOSONS}

For each point $\sigma \in \operatorname{DiffS}^{1} / S^{1}$ we have defined a Fock space $\mathcal{F}$. If we call $\mathcal{M}=\operatorname{Diff} S^{1} / S^{1}$ the base space we shall have the following bundle $X$ :

$$
\begin{aligned}
& \mathcal{F} \stackrel{i}{\rightarrow} X \\
& \downarrow \pi \text {. } \\
& M
\end{aligned}
$$

$\mathcal{F}$ is the fiber of the bundle $X$. In geometric language we define states of the theory as the constant sections of the bundle $X$. In other words, a state $\omega \in \mathcal{F}_{\sigma}$ (fiber $\mathcal{F}_{\text {in }}$ the point $\sigma \in \mathcal{M}$ ) represents the same state as $\omega \in \mathcal{F}_{\sigma}$ if $\sigma$ and $\omega$ are parallel. Up to now we have no means of comparing two vectors at different points of the base manifold. In order to do this we need to define a connection in such a way that the reparametrization invariance should be respected if possible. The bundle $\mathcal{F}$ has a vectorial subspace $V$ (the one-particle states) generated by a base of operators $\left\{T_{n}\right\}(n \in \mathbb{Z})$. A generic vector of $V$ is given by the linear combination

$$
v=v^{n} T_{n}, \quad v^{n} \in \mathbb{C} .
$$

Let us now introduce a vectorial bundle $\widetilde{X}$ with the same base as $X$ and fiber $V$ :

$$
\begin{array}{llll}
V & \stackrel{i}{\rightarrow} & \widetilde{X} & \\
& & \downarrow & \pi . \\
& & &
\end{array}
$$

For the bosonic field we have the expressions (2.14) or (2.15):

$$
\Pi^{[\sigma]}(\alpha)=\left(e_{[\sigma] n}^{b} \varphi^{[\sigma] n}\right) T_{b} .
$$

It is only natural to consider the bosonic field $\Pi^{[\sigma]}$ as a section of $\widetilde{X}$. Let us try to find a connection in $\widetilde{X}$ in such a way that $\Pi$ should be a constant section. This condition is given by

$$
\nabla_{\mathbf{x}} \Pi^{[\sigma]}=0 \text {, }
$$

where $\mathbf{X}$ belongs to tangent bundle $T(\mathcal{M})$. Equation (3.3) means that the bosonic field is reparametrization independent.

We shall treat $M$ and $T(M)$ in a simplified way following the analogy with the finite-dimensional case. Although this is not rigorous it furnishes satisfactory results.

Let $\left(q^{1}, \ldots, q^{n}\right)$ be a local coordinate system of a manifold $M$ and consider a bundle $A \approx M \times V$ locally, where $V$ is a vector space; $\omega_{i}$ being its base. A section of $A$ is

$$
S(q)=S^{i}(q) \omega_{i} .
$$

A local base for $T(M)$ is $\left(\partial / \partial q^{i}\right), i=1, \ldots, n$. The covariant derivative in the direction $\partial / \partial q_{i}$ is

$$
\left[\nabla_{\partial / \partial q} S(q)\right]^{j}=\frac{\partial S^{j}(q)}{\partial q^{i}}+\Gamma_{l j}^{i} S^{l}(q) .
$$

In our case the index $i$ is continuous and condition (3.3) is written as

$$
\frac{\delta}{\delta \sigma(\alpha)}\left(\frac{1}{\sqrt{2}} e_{[\sigma] n}^{m} \varphi^{[\sigma] n}\right)+\Gamma_{\alpha l}^{m}[\sigma]\left(\frac{1}{\sqrt{2}} e_{[\sigma] n}^{l} \varphi^{[\sigma] n}\right)=0
$$

or

$$
\frac{\delta}{\delta \sigma(\alpha)} e_{[\sigma] n}^{m}+e_{[\sigma] n}^{l} \Gamma_{\alpha l}^{m}=0
$$

This expression is the condition for zero torsion. By looking on the definition $(2.22)$ for the metric $\varrho^{[\sigma] m n}$ we 
see that $e_{[\sigma] n}^{m}$ are the vielbein. In conclusion we have arrived at a Riemannian geometry on $\mathcal{M}$ where $\Gamma_{\alpha l}^{m}$ is the spin connection.

Multiplying expression (3.4) by $e_{m}^{[\sigma] k}$, we obtain

$$
\Gamma_{\alpha k}^{m}=-e_{k}^{[\sigma] n} \frac{\delta}{\delta \sigma(\alpha)} e_{[\sigma] n}^{m}
$$

Recalling that

$\frac{\delta}{\delta \sigma(\alpha)} e_{[\sigma] n}^{m}=-\frac{i n}{L}\left(\frac{2 \pi}{L}\right) \exp \left[i \frac{2 \pi}{L}[m \alpha-n \sigma(\alpha)]\right)$

it follows that

$$
\Gamma_{\alpha k}^{m}=\frac{i k}{L}\left[\frac{2 \pi}{L}\right] \frac{1}{\eta(\alpha)} \exp \left[i \frac{2 \pi}{L}(k-m) \alpha\right] .
$$

Calculating now the curvature $R(X, Y)$ defined by

$$
\begin{aligned}
R(X, Y)_{c}^{b}= & {\left[\left(\nabla_{X}, \nabla_{Y}\right)-\nabla_{[X, Y]}\right] } \\
= & \frac{\delta}{\delta \sigma\left(\alpha^{\prime}\right)} \Gamma_{a c}^{b}-\frac{\delta}{\delta \sigma(\alpha)} \Gamma_{\alpha^{\prime} c}^{b} \\
& +\Gamma_{\alpha^{\prime} a}^{b} \Gamma_{\alpha c}^{a}-\Gamma_{\alpha a}^{b} \Gamma_{\alpha^{\prime} c}^{a},
\end{aligned}
$$

where $X=\delta / \delta \sigma\left(\alpha^{\prime}\right)$ and $Y=\delta / \delta \sigma(\alpha)$ belong to $T(M)$ and using (3.7) it follows that the right-hand side of (3.8) is zero (the detailed calculation is given in Appendix A).

The covariant derivative $\nabla$ given by $\Gamma_{\alpha c}^{a}$ which defines a parallel transport on $\widetilde{X}$ induces a covariant derivative $D_{\alpha}$ on $X$ through the equation

$$
\left\langle\Psi\left|e^{-i \epsilon D_{\alpha}}\left(e^{\epsilon \nabla_{x}} T_{k}\right) e^{i \epsilon D_{\alpha}}\right| \Phi\right\rangle=\left\langle\Psi\left|T_{k}\right| \Phi\right\rangle
$$

with $X=\delta / \delta \sigma(\alpha)$ and $D_{\alpha}^{\dagger}=D_{\alpha}$.

The parallel transport of operators $T_{k}$ defines the parallel transport of the states in such a way that the matrix elements should not change. The general form of $D_{\alpha}$ is given by

$$
D_{\alpha}=-i \frac{\delta}{\delta \sigma(\alpha)}+\Gamma_{[\sigma]}(\alpha)
$$

where $\Gamma(\alpha)$ is a Hermitian operator connection. If we expand in (3.9) up to first order in $\epsilon$ we have

$$
\left(I-i \epsilon D_{\alpha}\right)\left(I+\epsilon \nabla_{X}\right) T_{k}\left(I+i \epsilon D_{\alpha}\right)=T_{k} .
$$

That is

$$
\begin{aligned}
& i\left[D_{\alpha}, T_{k}\right]=\nabla_{X} T_{k}, \\
& {\left[\Gamma(\alpha), T_{k}\right]=-i \Gamma_{\alpha k}^{l} T_{l} .}
\end{aligned}
$$

The general form for $\Gamma(\alpha)$ which satisfies (3.12) is

$$
\Gamma_{[\sigma]}(\alpha)=B_{[\sigma]}^{m n}(\alpha) T_{m} T_{n}+f_{[\sigma]}(\alpha) .
$$

Introducing in (3.12), we obtain

$$
\Gamma_{[\sigma]}(\alpha)=\frac{1}{2 \eta(\alpha) L} \exp \left[\frac{2 \pi}{L}(l+k) \alpha\right) T_{l} T_{k}+f_{[\sigma]}(\alpha) \text {. }
$$

Using

$$
\Pi(\alpha)=T_{n} \varphi^{n}
$$

and taking the normal ordering in $\Gamma_{[\sigma]}(\alpha)$, we obtain

$$
\Gamma_{[\sigma]}(\alpha)=\frac{1}{2 \eta(\alpha)}: \Pi^{2}(\alpha):+f_{[\sigma]}(\alpha) .
$$

Calculating the curvature of $D_{\alpha}$

$$
\begin{aligned}
{\left[D_{\alpha}, D_{\alpha}\right]=} & -i \frac{\delta}{\delta \sigma(\alpha)} \Gamma\left(\alpha^{\prime}\right)+i \frac{\delta}{\delta \sigma\left(\alpha^{\prime}\right)} \Gamma(\alpha) \\
& +\left[\Gamma(\alpha), \Gamma\left(\alpha^{\prime}\right)\right] \\
= & F\left(\alpha, \alpha^{\prime}\right)-i \frac{\delta}{\delta \sigma(\alpha)} A\left(\alpha^{\prime}\right)+i \frac{\delta}{\delta \sigma\left(\alpha^{\prime}\right)} A(\alpha) \\
& +\left[A(\alpha), A\left(\alpha^{\prime}\right)\right]
\end{aligned}
$$

where

$F\left(\alpha, \alpha^{\prime}\right)=-i \frac{\delta}{\delta \sigma(\alpha)} f_{[\sigma]}\left(\alpha^{\prime}\right)+i \frac{\delta}{\delta \sigma\left(\alpha^{\prime}\right)} f_{[\sigma]}(\alpha)$,

$A(\alpha)=\frac{1}{2 \eta(\alpha)}: \Pi^{2}(\alpha):$

we have

$$
\begin{aligned}
& \frac{\delta}{\delta \sigma(\alpha)} \frac{1}{\eta\left(\alpha^{\prime}\right)}=-\partial_{\alpha^{\prime}} \delta\left(\alpha^{\prime}-\alpha\right) \frac{1}{\eta^{2}(\alpha)}, \\
& -i \frac{\delta}{\delta \sigma(\alpha)} A\left(\alpha^{\prime}\right)=i \partial_{\alpha^{\prime}} \delta\left(\alpha-\alpha^{\prime}\right) \frac{A\left(\alpha^{\prime}\right)}{\eta\left(\alpha^{\prime}\right)} .
\end{aligned}
$$

The algebra of $: \Pi^{2}$ : is the conformal algebra giving the result (see Appendix B)

$$
\begin{aligned}
{\left[\frac{: T^{2}(\alpha):}{2},\right.} & \left.\frac{: T^{2}\left(\alpha^{\prime}\right):}{2}\right] \\
= & i \partial_{\alpha} \delta\left(\alpha-\alpha^{\prime}\right)\left[\frac{: T^{2}(\alpha):}{2}+\frac{: T^{2}\left(\alpha^{\prime}\right):}{2}\right] \\
& -\frac{i}{24 \pi}\left[\delta^{\prime \prime \prime}\left(\alpha-\alpha^{\prime}\right)+\Delta\left[\frac{2 \pi}{L}\right)^{2} \delta^{\prime}\left(\alpha-\alpha^{\prime}\right)\right],
\end{aligned}
$$

where $\Delta=1$ for PBC's and $\Delta=-\frac{1}{2}$ for ABC's.

In Appendix $\mathrm{C}$ we give a discussion of the perturbative calculation of central term of the Virasoro algebra for the chiral bosons on the circle.

From expressions (3.16) and (3.20) it follows

$$
\begin{aligned}
{\left[D_{\alpha}, D_{\alpha^{\prime}}\right]=} & F\left(\alpha, \alpha^{\prime}\right)+\frac{-i}{24 \pi \eta(\alpha) \eta\left(\alpha^{\prime}\right)}\left[\delta^{\prime \prime \prime}\left(\alpha-\alpha^{\prime}\right)+\Delta\left[\frac{2 \pi}{L}\right]^{2} \delta^{\prime}(\alpha-\alpha)\right] \\
& +\partial_{\alpha} \delta\left(\alpha-\alpha^{\prime}\right)\left(\frac{A(\alpha)}{\eta\left(\alpha^{\prime}\right)}-\frac{A(\alpha)}{\eta(\alpha)}+\frac{A\left(\alpha^{\prime}\right)}{\eta(\alpha)}-\frac{A\left(\alpha^{\prime}\right)}{\eta\left(\alpha^{\prime}\right)}\right] .
\end{aligned}
$$


The last term in the right-hand side of (3.21) is zero. Since $f_{[\sigma]}(\alpha)$ in (3.15) is arbitrary you can choose it for each $\sigma$ in such a way that the curvature given by $D_{\alpha}$ is zero. In this way the curl of $f_{[\sigma]}(\alpha)$ is determined to be

$$
\begin{aligned}
F\left(\alpha, \alpha^{\prime}\right)=\frac{i}{24 \pi \eta(\alpha) \eta\left(\alpha^{\prime}\right)}[ & \delta^{\prime \prime \prime}\left(\alpha-\alpha^{\prime}\right) \\
& \left.+\Delta\left(\frac{2 \pi}{L}\right]^{2} \delta^{\prime}\left(\alpha-\alpha^{\prime}\right)\right] .
\end{aligned}
$$

Defining the adiabatic transport of the vacuum by

$$
D_{\alpha}^{\mathrm{ad}} \equiv\left\langle 0\left|D_{\alpha}\right| 0\right\rangle=-i \frac{\delta}{\delta \sigma(\alpha)}+f_{[\sigma]}(\alpha),
$$

which evidently does not create particles, the curvature of this operator is the right-hand side of (3.22):

$$
\left[D_{\alpha}^{\mathrm{ad}}, D_{\alpha^{\prime}}^{\mathrm{ad}}\right]=F\left(\alpha, \alpha^{\prime}\right) \text {. }
$$

This curvature is exactly the central term of the conformal algebra.

In order to understand better why we should use the adiabatic operator $D_{\alpha}^{\text {ad }}$ we will make the following considerations. We can obtain the vacuum state $|0, \sigma\rangle$ from $|0\rangle$ (the vacuum at the identity) by parallel transport that does not depend on the path. For instance we can consider a simple path $\gamma$ given by

$$
|0, \sigma\rangle \gamma=e^{i \epsilon D_{\alpha}}|0\rangle
$$

for some direction $\alpha$ in $M$.

The expectation value of the Hamiltonian operator is

$$
\begin{aligned}
\gamma\langle\sigma, 0|H| 0, \sigma\rangle \gamma & =\left\langle\sigma, 0\left|e^{-i \epsilon D_{\alpha}} H e^{i \epsilon D_{\alpha}}\right| 0, \sigma\right\rangle \\
& =\sum_{n}\left\langle 0\left|e^{-i \epsilon D_{\alpha}} H\right| n\right\rangle\left\langle n\left|e^{i \epsilon D_{\alpha}}\right| n\right\rangle \\
& =\sum_{n} E_{n}\left|\left\langle n\left|e^{i \epsilon D_{\alpha}}\right| n\right\rangle\right|^{2}
\end{aligned}
$$

where $|n\rangle$ is a complete set of eigenstates of $H$ : $H|n\rangle=E_{n}|n\rangle$.

Therefore, the parallel transport given by $D_{\alpha}$ has a problem: the vacuum energy depends on the parametrization $\sigma$. On the other hand, $D_{\alpha}$ has a nice property: it has zero curvature (no dependence on the path). From (3.10) and (3.15) it follows, to lowest order,

$$
\gamma\langle\sigma, 0|H| 0, \sigma\rangle^{\gamma} \simeq \frac{\epsilon^{2}}{4 \eta^{2}(\alpha)} \sum_{n} E_{n}\left|\left\langle n\left|: \Pi^{2}(\alpha):\right| n\right\rangle\right|^{2} \text {. }
$$

If we now consider $|0, \sigma\rangle_{\text {ad }}$ obtained from $|0\rangle$ by an adiabatic parallel transport generated by

$$
D_{\alpha}^{\mathrm{ad}}=D_{\alpha}-\frac{1}{2 \eta(\alpha)}: \Pi^{2}(\alpha)
$$

with

$$
|0, \sigma\rangle_{\mathrm{ad}}^{\gamma}=e^{i \epsilon D_{\alpha}^{\mathrm{ad}}}|0\rangle
$$

then it follows that ${ }_{\text {ad }}^{\gamma}\langle\sigma, 0|H| 0, \sigma\rangle$ ad $=0$ for any $\sigma$ in $M$.

In this case the curvature given by $D_{\alpha}^{\text {ad }}$ is different from zero and the vacuum $|0, \sigma\rangle\rangle_{\text {ad }}^{\gamma}$ now depends on the path $\gamma$. Therefore, the state $|0, \sigma\rangle \underset{\mathrm{ad}}{\gamma}$ is not well defined.

This proves that the choice of the adiabatic vacuum at each point of $M\left(\operatorname{Diff} S^{1} / S^{1}\right)$ introduces a curvature on the fiber bundle constructed on the basis $M$ with the special section in our case being the vacuum. This is related to the holonomy of the parallel transport of the vacuum, that is, the Berry phase. ${ }^{6}$ In other words, we are showing that the theory is not more invariant under the group of reparametrizations. Actually we have a projective representation of this group.

\section{ON THE HAMILTONIAN OF THE CHIRAL BOSONS IN AN EXTERNAL BACKGROUND}

Now we want to express the Hamiltonian given by (3.21):

$$
H=\frac{1}{2} \varrho^{[\sigma] a b} T_{[\sigma] a} T_{[\sigma] b}
$$

in terms of the bosonics fields. To this end recall the definition (3.22) of the curved metric and the expression (3.17) for the zweibein. In this way we arrive at

$H=\frac{1}{2} \int d \sigma \eta(\alpha) \Pi^{2}(\sigma(\alpha))=\frac{1}{2} \int d \sigma n(\sigma) \Pi^{2}(\sigma)$,

where we have defined $n(\sigma)=\eta(\alpha)$.

Bellucci, Gutterman, and Petcher ${ }^{4}$ have considered the action of a chiral boson interacting minimally with an external gravitational field described by

$$
S=\int d^{2} x e^{-1}\left[e_{+}^{\mu} \partial_{\mu} \phi e_{+}^{\mu} \partial_{\mu} \phi+\lambda^{--}\left(e_{-}^{\mu} \partial \phi\right)^{2}\right],
$$

where $e=\operatorname{det}\left(e_{a}^{\mu}\right), e_{ \pm}^{\mu}$ being the components of the zweibein and $\lambda^{-}$a Lagrange multiplier. After eliminating second-class constraints the auxiliary field $\lambda^{--}$gets fixed, arriving at expression (4.2) with

$$
n(\sigma)=\frac{e_{-}^{-}-e_{-}^{+}}{e_{-}^{-}+e_{-}^{+}} .
$$

This expression for the Hamiltonian is Lorentz and Weyl invariant. It has a residual static symmetry which comes from the original covariance of the action (4.3) under general coordinate transformations. This symmetry is given by

$$
\begin{aligned}
& \delta_{f} n(\sigma)=f(\sigma) \partial_{\sigma} n(\sigma)-n(\sigma) \partial_{\sigma} f(\sigma), \\
& \delta_{f} \phi(\sigma)=f(\sigma) \partial_{\sigma} \phi(\sigma) .
\end{aligned}
$$

The transformation of $n(\sigma)$ (4.5a) can be checked from (4.4) by using the static general transformation of a zweibein.

This symmetry can be realized in operator form with the generator of the bosonic field transformation given by

$$
\mathcal{L}_{f}=-\frac{1}{2} \int d \sigma f(\sigma) \Pi^{2}(\sigma) .
$$

Classically $\mathcal{L}_{f}$ is an element of the diffeomorphism algebra:

$$
\left[\mathcal{L}_{f}, \mathcal{L}_{g}\right]=i \mathcal{L}_{[f, g]} .
$$

Because of the quantization we must normal-order expression (4.6) obtaining a central term which comes from 
(3.20) if we recall that $: \Pi^{2}(\alpha): \alpha: T^{2}(\alpha):$. From (4.6) we obtain

$\mathcal{L}_{f}=\sum_{m, k=-\infty}^{\infty} \ell_{f}(k, m)$

$$
\begin{aligned}
& -\frac{1}{2} \theta\left(\omega_{m}\right) \theta\left(-\omega_{k}\right) a_{m} a_{-k-1} \\
& \left.-\frac{1}{2} \theta\left(-\omega_{m}\right) \theta\left(\omega_{k}\right) \frac{\delta}{\delta a_{-m-1}} \frac{\delta}{\delta a_{k}}\right),
\end{aligned}
$$

with

$$
\begin{aligned}
\ell_{f}(k, m)= & \frac{-\sqrt{\left|\omega_{k} \omega_{m}\right|}}{L} \\
& \times \int_{0}^{2 \pi} d \alpha \frac{f(\sigma)}{n^{2}(\sigma)} \exp \left[i\left(\omega_{m}-\omega_{k}\right) \xi(\sigma)\right] .
\end{aligned}
$$

Then it follows

$$
\left[\mathcal{L}_{f}, \mathcal{L}_{g}\right]=i \mathcal{L}_{[f, g]}+\mathrm{ST},
$$

where the Schwinger term (ST) is given by

$$
\begin{aligned}
\mathrm{ST}=\frac{1}{2} \sum_{k, m=-\infty}^{+\infty} \ell_{f}(m, k) \ell_{g}(k, m)[ & \theta\left(\omega_{k}\right) \theta\left(-\omega_{m}\right) \\
& \left.-\theta\left(\omega_{m}\right) \theta\left(-\omega_{k}\right)\right],
\end{aligned}
$$

Explicitly we have

$$
\begin{aligned}
\mathrm{ST}=\frac{-i}{24 \pi} \int_{0}^{2 \pi} d \sigma \frac{f(\sigma)}{n^{2}(\sigma)}[ & {\left[n(\sigma) \partial_{\sigma}\right]^{3} } \\
& \left.+\Delta\left(\frac{2 \pi}{L}\right)^{2} n(\sigma) \partial_{\sigma}\right) \frac{g(\sigma)}{n(\sigma)},
\end{aligned}
$$

where $\Delta=1$ for the periodic boundary condition and $\Delta=-\frac{1}{2}$ for the antiperiodic boundary condition.

\section{SEMENOFF-SANIELEVICI METHOD FOR CALCULATING THE CURVATURE FOR CHIRAL BOSONS ON THE CIRCLE}

Rewriting the Hamiltonian (4.2) in the form

$$
H=\frac{1}{2} \int d \sigma n(\sigma)\left[\partial_{\alpha} \phi(\alpha)\right]^{2}
$$

with

$$
\left[\phi(\alpha), \phi\left(\alpha^{\prime}\right)\right]=\frac{-i}{2} \epsilon\left(\alpha-\alpha^{\prime}\right),
$$

where $\epsilon\left(\alpha-\alpha^{\prime}\right)$ is given in (1.24), we can define the oneparticle Hamiltonian

$h \phi(\alpha)=i \partial_{\tau} \phi(\alpha)=[\phi(\alpha), H]=i n(\alpha) \partial_{\alpha} \phi(\alpha)$,

where the normalized eigenfunctions are given by

$$
\begin{aligned}
& h \phi_{n}(\alpha)=i n(\alpha) \partial_{\alpha} \phi_{n}(\alpha)=\omega_{n} \phi_{n}(\alpha), \\
& \phi_{n}(\alpha)=\frac{1}{\sqrt{L}} \exp \left[-i \omega_{n} \xi(\alpha)\right],
\end{aligned}
$$

where

$$
\begin{aligned}
& \xi(\alpha)=\int_{0}^{\alpha} \frac{d \alpha^{\prime}}{n\left(\alpha^{\prime}\right)}, \\
& \xi(2 \pi)=L, \\
& \omega_{n}=\frac{2 n \pi}{L}
\end{aligned}
$$

with $n \in \mathbb{Z}$ for periodic boundary conditions and $n \in \mathbb{Z}+\frac{1}{2}$ for antiperiodic boundary conditions. We want now to consider the dynamic effects of the external background field. For this reason we expand the secondquantized boson field $\phi(\alpha)$ in such a way that the Hamiltonian (5.1) has the usual diagonal form

$$
: H:=\sum_{k=0}^{\infty} \omega_{k} a_{k} \frac{\delta}{\delta a_{k}} \text {. }
$$

The expression which serves this purpose is

$$
\phi(\alpha)=\sum_{n=0}^{\infty^{\prime}}\left[\frac{\phi_{n}(\alpha)}{\sqrt{\omega_{n}}} \frac{\delta}{\delta a_{n}}+\frac{\phi_{n}^{*}(\alpha)}{\sqrt{\omega_{n}}} a_{n}\right)
$$

with $\phi_{n}(\alpha)$ defined in (5.5); the prime in the sum means that we exclude the singular contribution for $n=0$ in the periodic case. Using (5.2) it follows

$$
\left[\frac{\delta}{\delta_{k}}, a_{n}\right]=\delta_{n, m},
$$

as expected. In a very similar way as done by Sanielevici and Semenoff ${ }^{1}$ we introduce the operator of parallel transport $\Xi(\alpha)$ appropriate to the field (5.10):

$$
\Xi(\sigma)=\frac{1}{i} \frac{\delta}{\delta n(\sigma)}+\Omega(\sigma),
$$

which has zero curvature and commutes with (5.10).

Using $\left[\Xi(\sigma), \phi\left(\sigma^{\prime}\right)\right]=0$ we have

$$
\frac{1}{i} \frac{\delta}{\delta n(\sigma)} \phi\left(\alpha^{\prime}\right)=-\left[\Omega(\sigma), \phi\left(\sigma^{\prime}\right)\right]
$$

giving the solution

$$
\begin{aligned}
\Omega(\sigma)=\sum_{m, k=-\infty}^{\infty}\left\langle\omega_{n}\left|\frac{1}{i} \frac{\delta}{\delta n(\sigma)}\right| \omega_{m}\right\rangle & \mid \theta\left(\omega_{k}\right) \theta\left(\omega_{m}\right) a_{k} \frac{\delta}{\delta a_{m}}+\frac{1}{2} \theta\left(\omega_{k}\right) \theta\left(-\omega_{m}\right) a_{k} a_{-m-1} \\
& \left.-\frac{1}{2} \theta\left(-\omega_{k}\right) \theta\left(\omega_{m}\right) \frac{\delta}{\delta a_{-k-1}} \frac{\delta}{\delta a_{m}}\right]+\mathcal{A}(\sigma) .
\end{aligned}
$$

From the zero-curvature condition $\left[\Xi(\sigma), \Xi\left(\sigma^{\prime}\right)\right]=0$, we have 


$$
\frac{1}{i} \frac{\delta}{\delta n(\sigma)} \Omega\left(\sigma^{\prime}\right)-\frac{1}{i} \frac{\delta}{\delta n\left(\sigma^{\prime}\right)} \Omega(\sigma)=-\left[\Omega(\sigma), \Omega\left(\sigma^{\prime}\right)\right],
$$

which by use of (5.14) gives

$$
\begin{aligned}
\mathscr{F}_{S S}\left(\sigma, \sigma^{\prime}\right) & =\frac{1}{i} \frac{\delta}{\delta n(\sigma)} \mathcal{A}\left(\sigma^{\prime}\right)-\frac{1}{i} \frac{\delta}{\delta n\left(\sigma^{\prime}\right)} \mathcal{A}(\sigma) \\
& =-\frac{1}{2} \sum_{m, k=-\infty}^{\infty}\left\langle\omega_{k}\left|\frac{1}{i} \frac{\delta}{\delta n(\sigma)}\right| \omega_{m}\right\rangle\left\langle\omega_{m}\left|\frac{1}{i} \frac{\delta}{\delta n(\sigma)}\right| \omega_{k}\right\rangle\left[\theta\left(\omega_{k}\right) \theta\left(-\omega_{m}\right)-\theta\left(-\omega_{k}\right) \theta\left(\omega_{m}\right)\right]
\end{aligned}
$$

By definition we have

$$
\begin{aligned}
\left\langle\omega_{k}\left|\frac{1}{i} \frac{\delta}{\delta n(\sigma)}\right| \omega_{m}\right\rangle & =\left(\left.\frac{\left|\omega_{k}\right|}{\left|\omega_{m}\right|}\right|^{1 / 2} \int_{0}^{2 \pi} \frac{d \sigma^{\prime}}{n\left(\sigma^{\prime}\right)} \phi_{k}^{*}\left(\sigma^{\prime}\right) \frac{1}{i} \frac{\delta}{\delta n(\sigma)} \phi_{n}\left(\sigma^{\prime}\right)\right. \\
& =\operatorname{sgn} \omega_{n} \frac{\sqrt{\left|\omega_{k} \omega_{m}\right|}}{i L n^{2}(\sigma)} \frac{\exp \left[\left(\omega_{k}-\omega_{m}\right) \xi(\sigma)\right]}{\omega_{k}-\omega_{m}}
\end{aligned}
$$

for $m \neq k$. Substituting (5.17) in (5.16) it follows

$$
\begin{aligned}
\mathscr{F}_{S S}\left(\sigma, \sigma^{\prime}\right)=-\frac{1}{2 n^{2}(\sigma) n^{2}\left(\sigma^{\prime}\right) L^{2}} \sum_{k, m=-\infty}^{\infty} & \frac{\omega_{n} \omega_{k}}{\left(\omega_{k}-\omega_{n}\right)^{2}} \exp \left\{\left(\omega_{k}-\omega_{m}\right)\left[\xi(\sigma)-\xi\left(\sigma^{\prime}\right)\right]\right\} \\
& \times\left[\theta\left(\omega_{k}\right) \theta\left(-\omega_{m}\right)-\theta\left(-\omega_{k}\right) \theta\left(\omega_{m}\right)\right] .
\end{aligned}
$$

For the antiperiodic case (5.18) gives

$$
\begin{aligned}
\mathscr{F}_{S S}^{A}\left(\sigma, \sigma^{\prime}\right) & =-\frac{1}{8 n^{2}(\sigma) n^{2}\left(\sigma^{\prime}\right) L^{2}} \sum_{\substack{l=-\infty \\
l \neq 0}}^{\infty} \frac{1}{l^{2}} \exp \left[\frac{2 \pi i l}{L}\left[\xi(\sigma)-\xi\left(\sigma^{\prime}\right)\right]\right] \\
& \times \sum_{n=-\infty}^{+\infty}(2 m+1)(2 m+2 l+1)\left[\theta\left(\omega_{l+m}\right) \theta\left(-\omega_{m}\right)-\theta\left(-\omega_{l+m}\right) \theta\left(\omega_{m}\right)\right] \\
& =\left[\theta\left(\omega_{l-1}\right) \sum_{m=-l}^{-1}-\theta\left(-\omega_{l}\right) \sum_{m=0}^{-l-1}\right](2 m+1)(2 m+2 l+1)=\frac{-l\left(2 l^{2}+1\right)}{3}
\end{aligned}
$$

and therefore

$$
\begin{aligned}
\mathscr{F}_{S S}^{A}\left(\sigma, \sigma^{\prime}\right) & =-\frac{1}{8 n^{2}(\sigma) n^{2}\left(\sigma^{\prime}\right) L^{2}} \sum_{\substack{l=-\infty \\
l \neq 0}}^{\infty} \frac{2 l^{2}+1}{3 l} \exp \left[\frac{2 \pi i l}{L}\left[\xi(\sigma)-\xi\left(\sigma^{\prime}\right)\right]\right) \\
& =\frac{-i}{24 \pi n(\sigma) n\left(\sigma^{\prime}\right)} \partial_{\sigma} \delta\left(\sigma-\sigma^{\prime}\right)+\frac{i \pi}{24 n^{2}(\sigma) n^{2}\left(\sigma^{\prime}\right) L^{2}} \epsilon\left(\sigma-\sigma^{\prime}\right),
\end{aligned}
$$

where

$$
\epsilon\left(\sigma-\sigma^{\prime}\right)=\sum_{\substack{l=-\infty \\ l \neq 0}}^{\infty} \frac{1}{l} \exp \left[\frac{2 \pi i l}{L}\left[\xi(\sigma)-\xi\left(\sigma^{\prime}\right)\right]\right] .
$$

For the periodic case in (5.18) we should take $k \neq 0, m \neq 0$ and we obtain

$$
\begin{aligned}
\mathcal{F}_{S S}^{P}\left(\sigma, \sigma^{\prime}\right)=-\frac{1}{8 n^{2}(\sigma) n^{2}\left(\sigma^{\prime}\right) L^{2}} \sum_{l=-\infty}^{+\infty} & \frac{1}{l^{2}} \exp \left[\frac{2 \pi i l}{L}\left[\xi(\sigma)-\xi\left(\sigma^{\prime}\right)\right]\right) \\
& \times \sum_{\substack{m=-\infty \\
m \neq 0}}^{\infty} 2 m(2 m+2 l)\left[\theta\left(\omega_{l+m}\right) \theta\left(-\omega_{m}\right)-\theta\left(-\omega_{l+m}\right) \theta\left(\omega_{m}\right)\right]
\end{aligned}
$$

and using

$$
\left[\theta\left(\omega_{l-1}\right) \sum_{m=-l+1}^{-1}-\theta\left(-\omega_{l}\right) \sum_{m=1}^{-l-1}\right] 4\left(m^{2}+m l\right)=-\frac{2}{3} l\left(l^{2}-1\right)
$$


expression (5.22) can be rewritten as

$$
\begin{aligned}
\mathcal{F}_{S S}^{P}\left(\sigma, \sigma^{\prime}\right) & =-\frac{1}{8 n^{2}(\sigma) n^{2}\left(\sigma^{\prime}\right) L^{2}} \sum_{l=-\infty}^{+\infty} \frac{2 l^{2}+1}{l} \exp \left(\frac{2 \pi i l}{L}\left[\xi(\sigma)-\xi\left(\sigma^{\prime}\right)\right]\right) \\
& =\frac{-i}{24 \pi n(\sigma) n\left(\sigma^{\prime}\right)} \partial_{\sigma} \delta\left(\sigma-\sigma^{\prime}\right)-\frac{i \pi}{12 n^{2}(\sigma) n^{2}\left(\sigma^{\prime}\right) L^{2}} \epsilon\left(\sigma-\sigma^{\prime}\right) .
\end{aligned}
$$

We now want to see the relation between the curvatures $\mathscr{F}_{S S}$ given in (5.20) and (5.24) and $F$ given in (3.22) obtained by geometrical considerations. We have mentioned in the preceding section that the Hamiltonian (4.2) is invariant under the static transformations (4.5). The transformation (4.5),

$\delta_{f} n(\alpha)=f(\alpha) \partial_{\alpha} n(\alpha)-n(\alpha) \partial_{\alpha} f(\alpha)=(f, n)(\alpha)$,

closes a Lie algebra:

$$
\delta_{f} \delta_{g}-\delta_{g} \delta_{f}=-\delta_{(f, g)} n(\alpha) .
$$

In the theory of conformal quantum fields a continuous symmetry is given by the exponentiation of an Hermitian operator. Classically the active transformation on the bosonic fields generated by $\mathcal{L}_{f}$ of expression (4.6) is equivalent to a passive transformation of the "metric" $n(\alpha)$. Since $\Xi(\alpha)$ introduced in (5.12) commutes with the field $\phi(\alpha)$, we realize quantically the symmetry given by (5.25) by the exponentiation of the following infinitesimal Hermitian generator:

$$
T_{f}=\int d \alpha(f, n) \Xi(\alpha)
$$

with the property

$$
\left[n(\alpha), T_{f}\right]=i \delta_{f} n(\alpha) .
$$

The operators $T_{f}$ satisfy the algebra

$$
\left[T_{f}, T_{g}\right]=i T_{(f, g)} \text {. }
$$

So we have a faithful representation of the algebra (5.25) and (5.26). The representation of the group can be shown to be unitary. Using expressions (5.27) and (4.6) it holds that

$$
\left[T_{f}, \mathcal{L}_{g}\right]=\mathrm{ST}
$$

for $g(\alpha)$ independent of $n(\alpha)$ and ST given by expression (4.12). Instead for $g(\alpha)=n(\alpha)$ we have

$$
\left[T_{f}, \mathcal{L}_{n}\right]=\left[T_{f}, H\right]=-i \mathcal{L}_{(f, n)}
$$

and therefore $T_{f}$ is not a symmetry of the Hamiltonian. Defining the adiabatic operator

$$
T_{f}^{\mathrm{ad}}=T_{f}+\mathcal{L}_{f}
$$

using (5.32) and (4.6) we have

$$
\left[T_{f}^{\mathrm{ad}}, \mathcal{L}_{n}\right]=\left[T_{f}^{\mathrm{ad}}, H\right]=0 \text {, }
$$

which therefore explains the adiabatic nature of (5.32). Using (5.27) and (4.6) we have

$$
\begin{aligned}
T_{f}^{\mathrm{ad}} & =\int_{0}^{2 \pi} d \alpha(f, n) \Xi^{\mathrm{ad}}(\alpha) \\
& =\int_{0}^{2 \pi} d \alpha(f, n)\left[\frac{1}{i} \frac{\delta}{\delta n(\sigma)}+\mathcal{A}(\sigma)\right)
\end{aligned}
$$

with $\mathcal{A}(\alpha)$ introduced in (5.14). The algebra of $T_{f}^{\mathrm{ad}}$ is easily calculated as

$$
\begin{aligned}
{\left[T_{f}^{\mathrm{ad}}, T_{g}^{\mathrm{ad}}\right]=} & i T_{(f, g)}^{\mathrm{ad}}+\int^{2 \pi} d \alpha \int_{0}^{2 \pi} d \alpha^{\prime}(f, g)(\alpha) \mathcal{F}_{S S} \\
& \times\left(\alpha, \alpha^{\prime}\right)(g, n)(\alpha) \\
= & i T_{(f, g)}^{\mathrm{ad}}-\mathrm{ST} .
\end{aligned}
$$

Now according to $(4.12)$ we can rewrite ST in the form

$$
\begin{aligned}
\text { ST } & =\frac{-i}{24 \pi} \int_{0}^{2 \pi} \frac{d \xi(\sigma)}{n(\sigma)} f(\sigma)\left[\left(\frac{\partial}{\partial \xi(\sigma)}\right]^{2}+\Delta\left[\frac{2 \pi}{L}\right]^{2} \frac{\partial}{\partial \xi(\sigma)}\right] \frac{g(\sigma)}{n(\sigma)} \\
& =\frac{-i}{24 \pi} \int_{0}^{2 \pi} \frac{d u}{n(\sigma)} f(\sigma)\left[\left[\frac{\partial}{\partial u}\right]^{3}+\Delta\left[\frac{2 \pi}{L}\right]^{2} \frac{\partial}{\partial u}\right] \frac{g(\sigma)}{n(\sigma)} \\
& =\frac{-i}{24 \pi} \int_{0}^{2 \pi} d u \int_{0}^{2 \pi} d u^{\prime} \frac{f\left(\sigma^{\prime}\right)}{n\left(\sigma^{\prime}\right)} \delta\left(u-u^{\prime}\right)\left[\frac{\partial^{3}}{\partial u^{3}}+\Delta\left[\frac{2 \pi}{L}\right]^{2} \frac{\partial}{\partial u}\right] \frac{g(\sigma)}{n(\sigma)} \\
& =\frac{-i}{24 \pi} \int_{0}^{2 \pi} d u \int_{0}^{2 \pi} d u^{\prime}\left\{\left[\frac{\partial^{3}}{\partial u^{3}}+\Delta\left[\frac{2 \pi}{L}\right]^{2} \frac{\partial}{\partial u}\right] \delta\left(u-u^{\prime}\right)\right\} \frac{f\left(\sigma^{\prime}\right) g(\sigma)}{n\left(\sigma^{\prime}\right) n(\sigma)}
\end{aligned}
$$

Now

$$
\eta(u)=\frac{\partial \sigma}{\partial u}=n(\sigma)
$$

and therefore (5.36) reproduces the curvature (3.22).
Comparing with the calculation of Ref. 3 with WeylMajorana fermions, we have obtained, as expected, the double value for the central term, but with the opposite boundary conditions. 


\section{CONCLUSION}

In conclusion, we have obtained a generalized version of the Floreanini-Jackiw Lagrangian starting from a chiral constrained real boson. We quantized and interpreted the states as sections of a bundle over DiffS $S^{1} / S^{1}$. First, we have a natural parallel transport of the bosonic operator basis by the reparametrization transformation of the bosonic field. In this way it follows a covariant derivative with zero curvature. The parallel transport of the bosonic operator induces a parallel transport of the states (3.9). Imposing a zero curvature for this new derivative we observe that the vacuum energy is not preserved along the transport. But if we do not want this we have to use the adiabatic transport which does not have a zero curvature (reparametrization anomaly). In Appendix $C$ we verified that this anomaly is the same that is obtained by perturbation calculation. We have used two approaches to the problem of the anomaly: using reparametrizations and using a background field. In the last one it is explicitly the interaction with the background field, but both are equivalent. It is also verified that the chiral boson is equivalent (with opposite boundary conditions) to the Weyl fermion obtaining the usual equivalence between bosonic and fermionic partition functions. $^{7}$

\section{ACKNOWLEDGMENTS}

The work of J.R.R.M. was supported by Coordenação de Aperfeiçoamento de Pessoal do Ensino Superior, Brazil. The work of A.H.Z. was partially supported by Conselho Nacional de Desenvolvimento Científico e Tecnológico, Brazil. The work of P.T.S. was supported by Fundação de Amparo e Pesquisa do Estado de São Paulo, Brazil.

\section{APPENDIX A: CALCULATION OF THE CURVATURE (3.8)}

Let us check that $R(X, Y)_{c}^{b}$ given in (3.8) is zero. We have

$$
\begin{gathered}
\frac{\delta}{\delta \sigma\left(\alpha^{\prime}\right)}\left[\frac{i c}{\eta(\alpha) L}\left[\frac{2 \pi}{L}\right] \exp \left[i \frac{2 \pi}{L}(b-c) \alpha\right]\right] \\
=\frac{i c}{L}\left[\frac{2 \pi}{L}\right] \exp \left[i \frac{2 \pi}{L}(b-c) \alpha\right] \frac{\delta}{\delta \sigma\left(\alpha^{\prime}\right)} \frac{1}{\eta(\alpha)} .
\end{gathered}
$$

Now

$$
\frac{\delta}{\delta \sigma\left(\alpha^{\prime}\right)}=\int d x \frac{\delta \eta(x)}{\delta \sigma\left(\alpha^{\prime}\right)} \frac{\delta}{\delta \sigma(x)}
$$

and therefore

$$
\frac{\delta}{\delta \sigma\left(\alpha^{\prime}\right)} \frac{1}{\eta(\alpha)}=-\partial_{\alpha^{\prime}} \delta\left(\alpha-\alpha^{\prime}\right) \frac{1}{\eta^{2}(\alpha)} \text {. }
$$

Therefore,

$$
\begin{aligned}
\frac{\delta}{\delta \sigma\left(\alpha^{\prime}\right)} \Gamma_{\alpha c}^{b}= & \frac{-i c}{L}\left[\frac{2 \pi}{L}\right] \frac{\exp \left[i \frac{2 \pi}{L}(b-c) \alpha\right]}{\eta^{2}(\alpha)} \\
& \times \partial_{\alpha} \delta\left(\alpha-\alpha^{\prime}\right) .
\end{aligned}
$$

Also we have

$$
\begin{aligned}
& \Gamma_{\alpha^{\prime} \alpha}^{b} \Gamma_{\alpha c}^{a}=\sum_{a} \frac{i a}{L}\left(\frac{2 \pi}{L}\right) \frac{\exp \left[i \frac{2 \pi}{L}(b-a) \alpha^{\prime}\right]}{\eta\left(\alpha^{\prime}\right)} \\
& \times \frac{i c}{L}\left(\frac{2 \pi}{L}\right) \frac{\exp \left[i \frac{2 \pi}{L}(b-c) \alpha\right]}{\eta(\alpha)}
\end{aligned}
$$

or

$$
\begin{aligned}
\Gamma_{\alpha^{\prime} a}^{b} \Gamma_{\alpha c}^{a}= & \frac{i c}{L}\left(\frac{2 \pi}{L}\right) \frac{\exp \left[i \frac{2 \pi}{L}\left(b \alpha^{\prime}-c \alpha\right)\right]}{\eta(\alpha) \eta\left(\alpha^{\prime}\right)} \\
& \times \partial_{\alpha} \delta\left(\alpha-\alpha^{\prime}\right) .
\end{aligned}
$$

From (B1) and (B2), the result (3.8) follows.

\section{APPENDIX B: CALCULATION OF EXPRESSION (3.20)}

Let us consider $T(\alpha)$ given by (3.14). The conformal algebra is generated by $T^{2}(\alpha) / 2$ which is given by

$$
\begin{aligned}
\frac{: T^{2}(\alpha):}{2} & =\frac{1}{L}\left[\frac{2 \pi}{L}\right]: \sum_{n}\left[\frac{1}{2} \sum_{l} \widetilde{T}_{l} \widetilde{T}_{n-l}\right]: \exp \left[i \frac{2 \pi}{L} n \alpha\right) \\
& =\frac{1}{L}\left(\frac{2 \pi}{L}\right] \sum_{n}: L_{-n}: \exp \left[i \frac{2 \pi}{L} n \alpha\right),
\end{aligned}
$$

where we have introduced

$$
\widetilde{T}_{n}=\left(\frac{L}{2 \pi}\right)^{1 / 2} T_{n}
$$

satisfying

$$
\left[\widetilde{T}_{n}, \widetilde{T}_{m}\right]=n \delta_{n+m, 0}
$$

and

$$
L_{n}=\frac{1}{2}: \sum_{l} \widetilde{T}_{l} \widetilde{T}_{-n-l}:
$$

being the usual Virasoro generators satisfying

$$
\left[L_{n}, L_{m}\right]=(n-m) L_{n+m}+\delta_{n+m, 0} \frac{1}{12}\left(n^{3}-\Delta n\right),
$$

where $\Delta=1$ for PBC's and $\Delta=-\frac{1}{2}$ for ABC's. Therefore,

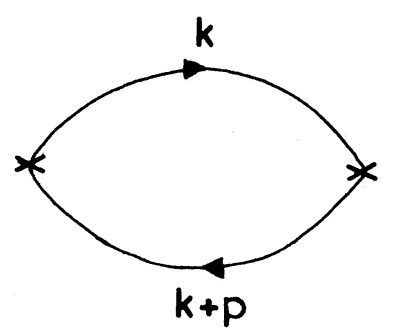

FIG. 2. One-loop diagram corresponding to $\langle 0| T\{[\mathcal{H}(\sigma, \tau)$, $\left.\left.\mathscr{H}\left(\sigma^{\prime}, \tau^{\prime}\right)\right]\right\}|0\rangle$. 


$$
\begin{aligned}
{\left[\frac{: T^{2}(\alpha):}{2}, \frac{: T^{2}\left(\alpha^{\prime}\right):}{2}\right] } & =\left[-\frac{1}{L} \frac{2 \pi}{L}\right]^{2} \sum_{m n}\left[L_{-n}, L_{-m}\right] \exp \left[i \frac{2 \pi}{L}\left(m \alpha^{\prime}+n \alpha\right)\right) \\
& =i \partial_{\alpha} \delta\left(\alpha-\alpha^{\prime}\right)\left(\frac{: T^{2}(\alpha):}{2}+\frac{: T^{2}\left(\alpha^{\prime}\right):}{2}\right)+\frac{-i}{24 \pi}\left[\delta^{\prime \prime \prime}\left(\alpha-\alpha^{\prime}\right)+\Delta\left(\frac{2 \pi}{L}\right]^{2} \delta^{\prime}\left(\alpha-\alpha^{\prime}\right)\right],
\end{aligned}
$$

where we have used (B5).

\section{APPENDIX C: PERTURBATIVE CALCULATION OF THE CENTRAL TERM OF VIRASORO ALGEBRA FOR THE CHIRAL BOSONS ON A CIRCLE}

In this appendix we calculate the two-point function of the energy-momentum tensor corresponding to the Lagrangian

$$
\mathcal{L}=\frac{1}{2}\left(\dot{\phi} \phi^{\prime}-\phi^{\prime 2}\right)
$$

with the Hamiltonian density $\mathcal{H}=\frac{1}{2} \phi^{\prime 2}$.

Let us obtain the algebra $\left[\mathscr{H}(\sigma), \mathscr{H}\left(\alpha^{\prime}\right)\right]$ by using a trick that is well known in current algebra.
Consider the time-ordered, two-point function $\left\langle 0\left|T\left\langle\left[\mathscr{H}(\sigma), \mathscr{H}\left(\sigma^{\prime}\right)\right]\right\rangle\right| 0\right\rangle$. It is not conserved but obeys the Ward-type identity

$$
\begin{aligned}
\partial_{-}\left\langle 0\left|T\left\{\left[\mathcal{H}(\sigma, \tau), \mathscr{H}\left(\sigma^{\prime}, \tau^{\prime}\right)\right]\right\}\right| 0\right\rangle \\
\quad=\frac{1}{\sqrt{2}} \delta\left(\tau-\tau^{\prime}\right)\left\langle 0\left|T\left\{\left[\mathcal{H}(\sigma), \mathscr{H}\left(\sigma^{\prime}\right)\right]\right\}\right| 0\right\rangle ;
\end{aligned}
$$

with this expression we calculate only the anomaly of the commutator. The diagram which corresponds to

$$
\left\langle 0\left|T\left\{\left[\mathcal{H}(\sigma, \tau), \mathscr{H}\left(\sigma^{\prime}, \tau^{\prime}\right)\right]\right\}\right| 0\right\rangle
$$

given the value

$$
\frac{2 \pi}{L} \frac{2}{4} \int \frac{d k_{t}}{(2 \pi)^{2}} \sum_{n=-\infty}^{+\infty} \frac{k_{x}\left(k_{x}+p_{x}\right)}{\left[k_{t}-k_{x}+\frac{i \epsilon}{k_{x}}\right]\left[k_{x}+p_{t}-k_{x}-p_{x}+\frac{i \epsilon}{k_{x}+p}\right]}
$$

corresponding to the diagram of Fig. 2.

We have $k_{x}=2 n \pi / L ; p_{x}=2 m \pi / L$ with $n, m \in \mathbb{Z}$ for the periodic boundary conditions and $n, m \in \mathbb{Z}+\frac{1}{2}$ for the antiperiodic boundary conditions.

Integrating by residues in $k_{t}$ expression (C3) gives

$$
\begin{aligned}
\frac{+i}{2 L} \sum_{n=1-m}^{0} \frac{k_{x}\left(k_{x}+p_{x}\right)}{p_{x}-p_{t}} & \\
& =\frac{-i}{24 \pi} \frac{1}{p_{x}+p_{t}}\left[p_{x}^{3}-\Delta\left(\frac{2 \pi}{L}\right)^{2} p_{x}\right],
\end{aligned}
$$

where $\Delta=1$ for PBC's and $\Delta=-\frac{1}{2}$ for ABC's.
Introducing expression (C2) and taking the Fourier transform of (C4) through the rules $p_{+} \rightarrow i \partial_{+}, p_{-} \rightarrow i \partial_{-}$, $1 \rightarrow \delta\left(\sigma-\sigma^{\prime}\right) \delta\left(\tau-\tau^{\prime}\right)$ with our convention that $p_{ \pm}=(1 / \sqrt{2})\left(p_{t} \pm p_{x}\right)$ the usual result follows:

$$
\begin{aligned}
&\langle 0| T\left\{\left[\mathcal{H}(\sigma, \tau), \mathcal{H}\left(\sigma^{\prime}, \tau\right)\right]\right\}|0\rangle \\
& \quad=\frac{-i}{24 \pi}-\left[\delta^{\prime \prime \prime}\left(\alpha-\alpha^{\prime}\right)+\Delta\left[\frac{2 \pi}{L}\right]^{2} \delta^{\prime}\left(\alpha-\alpha^{\prime}\right)\right] .
\end{aligned}
$$

The second term can be absorbed in a redefinition of H. It corresponds to the zero-point energy (Casimir effect).
${ }^{1}$ M. N. Sanielevici and G. W. Semenoff, Phys. Rev. D 37, 2934 (1988); Phys. Lett. B 198, 209 (1987).

${ }^{2}$ M. J. Bowick and S. G. Rajeev, Phys. Rev. Lett. 58, 535 (1987); Nucl. Phys. B293, 348 (1987).

${ }^{3}$ R. Floreanini and R. Jackiw, Phys. Rev. Lett. 59, 1873 (1987);

M. E. V. Costa and H. O. Girotti, ibid. 60, 1771 (1988).

${ }^{4}$ S. Bellucci, M. F. L. Golterman, and D. N. Petcher, Report
No. UCD-88-19, 1988 (unpublished).

${ }^{5}$ P. A. M. Dirac, Lectures on Quantum Mechanics (Academic, New York, 1987).

${ }^{6}$ M. V. Berry, Proc. R. Soc. London A392, 45 (1984); B. Simon, Phys. Rev. Lett. 51, 2167 (1983).

${ }^{7}$ M. B. Green, J. Schwarz, and E. Witten, Superstring Theory (Cambridge University Press, Cambridge, England, 1987). 